\title{
Effects of pulmonary artery banding in doxorubicin-induced left ventricular cardiomyopathy
}

Can Yerebakan, MD, ${ }^{\mathrm{a}}$ Johannes Boltze, MD, PhD,${ }^{\mathrm{b}}$ Hatem Elmontaser, MD, ${ }^{\mathrm{c}}$ Uygar Yoruker, MD, Heiner Latus, MD, ${ }^{\mathrm{c}}$ Markus Khalil, MD ${ }^{\mathrm{c}}$ Stefan Ostermayer, MD, ${ }^{\mathrm{d}}$ Blanca Steinbrenner, MD, Christian Apitz, MD, ${ }^{\mathrm{e}}$ Matthias Schneider, DVM, ${ }^{\mathrm{f}}$ Marcel Suchowski, DVM,${ }^{\mathrm{g}}$ Rita Ruetten, DVM, PhD, ${ }^{\mathrm{h}}$ Kristin Mueller, DVM, PhD, ${ }^{\mathrm{g}}$ Gunther Kerst, MD, ${ }^{\mathrm{d}}$ Dietmar Schranz, MD, ${ }^{\mathrm{c}}$ and Hakan Akintuerk, $\mathrm{MD}^{\mathrm{c}}$

\section{ABSTRACT}

Objective: Central pulmonary banding has been proposed as a novel alternative for the treatment of left ventricular dilated cardiomyopathy in children. We sought to investigate the effects of central pulmonary banding in an experimental model of doxorubicin-induced left ventricular dilated cardiomyopathy.

Methods: Four-month-old sheep $(\mathrm{n}=28)$ were treated with intermittent intracoronary injections of doxorubicin $(0.75 \mathrm{mg} / \mathrm{kg} / \mathrm{dose})$ into the left main coronary artery. A total dose of up to $2.15 \mathrm{mg} / \mathrm{kg}$ of doxorubicin was administered until signs of left ventricular dilation with functional impairment occurred by transthoracic echocardiography evaluation. Animals that survived were treated with surgical central pulmonary banding through a left anterior thoracotomy or sham surgery. Transthoracic echocardiography and pressure-volume loop measurements were used to compare left ventricular function preoperatively and 3 months later. Macroscopic and microscopic histologic examinations followed after hearts were harvested.

Results: Nine animals from the central pulmonary banding group and 8 animals from the sham group survived and were included in the final analysis. Both groups showed similar inflammation and fibrosis upon histologic examination consistent with the toxic myocardial effects of doxorubicin. There were no differences in the echocardiographic measurements before central pulmonary banding or sham operation. Baseline measurements before the central pulmonary banding/sham operation were considered as $100 \%$. The central pulmonary banding group had better left ventricular ejection fraction $(102.5 \% \pm 21.6 \%$ vs $76.7 \% \pm 11.7 \%$, $P=.01)$, with a tendency for smaller left ventricular end-diastolic $(101.2 \% \pm 7.4 \%$ vs $120.4 \% \pm 10.8 \%, P=.18)$ and significantly smaller endsystolic $(100.3 \% \pm 12.9 \%$ vs $116.5 \pm 9.6 \%, P=.02)$ diameter of the left ventricle in comparison with the sham animals at 3 months. The end-systolic volume $(101.4 \% \pm 31.6 \%$ vs $143.4 \% \pm 28.6 \%, P=.02)$ was significantly lower in the central pulmonary banding group 3 months postoperatively. Fractional shortening in the long axis $(118.5 \% \pm 21.5 \%$ vs $85.2 \% \pm 22.8 \%, P=.016)$ and short axis $(122.5 \% \pm 18 \%$ vs $80.9 \% \pm 13.6 \%, P=.0005)$ revealed significantly higher values in the central pulmonary banding group. In the conductance catheter measurements, no significant differences were seen between the groups for the parameters of systolic and diastolic function.

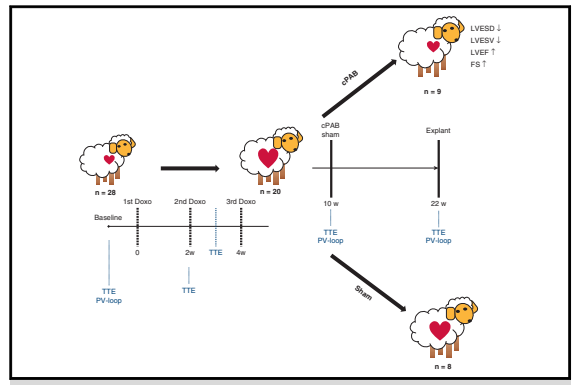

Effects of CPAB in toxic left ventricular cardiomyopathy.

Central Message

cPAB in the setting of experimental toxic left ventricular dilated cardiomyopathy improved left ventricular echocardiographic function and dimensions.

\section{Perspective}

cPAB was proposed as an alternative treatment for left ventricular dilated cardiomyopathy in infants and young children with beneficial effects on left ventricular function. This simple technique may be used for further causes that lead to left ventricular dysfunction with spatial dilation

See Commentaries on pages 2429 and 2431.

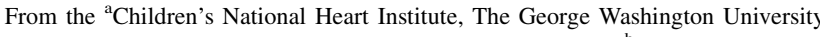
School of Medicine and Health Sciences, Washington, DC; ${ }^{b}$ School of Life Sciences, University of Warwick, Coventry, United Kingdom; ${ }^{\mathrm{c} P e d i a t r i c ~ H e a r t ~ C e n t e r ~}$ Giessen, Justus-Liebig-University, Giessen, Germany; ${ }^{\mathrm{d} D e p a r t m e n t}$ of Pediatric Cardiology, University of Aachen, Aachen, Germany; ${ }^{\mathrm{e} P e d i a t r i c ~ C a r d i o l o g y, ~ U n i-~}$ versity of Ulm, Ulm, Germany; ${ }^{f}$ Veterinary Medicine Clinic for Small Animals, Justus-Liebig-University, Giessen, Germany; ${ }^{\mathrm{g}}$ Institute of Pathology, Faculty of Veterinary Medicine, University Leipzig, Leipzig, Germany; and hraunhofer-Institute for Cell Therapy and Immunology, Leipzig, Germany.

Funded by "Fördergemeinschaft Deutsche Kinderherzzentren e.V" Germany.
}

Read at the 98th Annual Meeting of The American Association for Thoracic Sur gery, San Diego, California, April 28-May 1, 2018.

Received for publication May 2, 2018; revisions received Dec 21, 2018; accepted for publication Jan 14, 2019; available ahead of print April 8, 2019.

Address for reprints: Can Yerebakan, MD, Department of Cardiovascular Surgery, Children's National Heart Institute, The George Washington University School of Medicine and Health Sciences, 111 Michigan Ave NW, Washington, DC 20010 (E-mail: cyerebakan@childrensnational.org).

$0022-5223 / \$ 36.00$

Copyright (C) 2019 by The American Association for Thoracic Surgery

https://doi.org/10.1016/j.jtcvs.2019.01.138 


\section{Abbreviations and Acronyms}

$\mathrm{cPAB}=$ central pulmonary artery banding

$\mathrm{dp} / \mathrm{dt}_{\max }=$ maximal slope of systolic pressure increment

$\mathrm{dp} / \mathrm{dt}_{\min }=$ maximal slope of diastolic pressure decrement

LVEF = left ventricular ejection fraction

$\mathrm{PAB}=$ pulmonary artery banding

$\mathrm{PV} \quad=$ pressure-volume

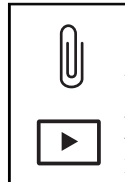

Scanning this QR code will take you to the article title page to access supplementary information. To view the AATS Annual Meeting Webcast, see the URL next to the webcast thumbnail.

Conclusions: Central pulmonary artery banding in the setting of experimental toxic left ventricular dilated cardiomyopathy improved left ventricular echocardiographic function and dimensions. ( $\mathrm{J}$ Thorac Cardiovasc Surg 2019;157:2416-28)

Cardiomyopathies in the pediatric population have an overall incidence of 10 to 12 cases per million. The most common form in children is dilated cardiomyopathy, which occurs in more than $50 \%$ of all cardiomyopathies in childhood. ${ }^{1,2}$ Transplant-free survival in these patients at 1 year and 5 years is reported to be approximately $70 \%$ and $60 \%$, respectively. ${ }^{3,4}$

The clinical presentation of dilated cardiomyopathy is primarily a dilated left ventricular chamber with severely impaired function on echocardiographic evaluation. The underlying etiology may be acute or chronic myocarditis, metabolic or neuromuscular disorders, or noncompaction or idiopathic cardiomyopathy. ${ }^{2,3,5}$ Left ventricular dysfunction and dilation are usually accompanied by a mild to moderate degree of mitral regurgitation in the early phase. The right ventricle is mostly spared and exhibits milder dysfunction. ${ }^{6}$

Although conservative medical heart failure therapy may be initiated, further decline in left ventricular function is not rare, and end-stage heart failure may ensue rapidly, requiring mechanical ventilation and inotropic support. However, in severe cases mechanical circulatory support or ultimately heart transplantation will be needed. Mechanical circulatory support for children as a bridge-to-heart transplantation bears substantial potential for severe complications, such as coagulation disorder, embolic insult, infection, and death. ${ }^{7}$ Studies continue to determine solutions for the optimal circulatory assistance in the pediatric population. Cardiac transplantation remains the gold standard therapy for end-stage heart failure in the pediatric population. However, the chronic shortage of donor organs hinders a wider application of heart transplantation. Even after a successful heart transplant, the 5-year survival remains at $60 \%{ }^{8}$ Alternative therapies for the treatment of childhood cardiomyopathy are warranted.

Inspired by the advantageous effects of central pulmonary artery banding (cPAB) for the palliation of 1-transposition of the great arteries, the first case report of $\mathrm{CPAB}$ in a child with left ventricular cardiomyopathy was published by the Giessen group in $2007 .^{9}$ The positive midterm results of 12 patients were reported by Schranz and colleagues in 2013. ${ }^{10}$ The experience with cPAB for left ventricular cardiomyopathy has been reported with promising outcomes in 70 patients worldwide. ${ }^{11}$ The basis for this approach is treatment of left ventricular cardiomyopathy using $\mathrm{CPAB}$, which leads to pressure overload of the right ventricle that is required to possess near to normal function. An improvement of left ventricular function is observed in most treated children within several months. The underlying mechanism for the improvement of left ventricular function is still unknown. The aim of this experimental study was to investigate the myocardial functional and left ventricular dimensional changes using cPAB in a doxorubicin-induced toxic left ventricular cardiomyopathy in a validated model. ${ }^{12}$

\section{MATERIALS AND METHODS}

\section{Animals and Anesthesia}

Animals received humane care in compliance with the Principles of Laboratory Animal Care formulated by the National Society for Medical Research and the Guide for the Care and Use of Laboratory Animals in Germany. The study was approved by the authority for animal protection in Hessen, Germany (V54-19c 20/15 - FU/1023).

This study was carried out with the use of 30 four-month-old domestic sheep (median weight, $29 \mathrm{~kg}$; range, 29-36 kg). Two animals were excluded before the start of the study because of other illnesses.

\section{Anesthesia and Mechanical Ventilation}

All animals received a 10 to $20 \mathrm{mg} / \mathrm{kg}$ ketamine dose, followed by intravenous access through an ear vein. Intubation and mechanical ventilation (Excel 210 SE, Ohnmeda-BOC Group, Madison, Wis) were established. Propofol infusion was used for maintenance along with anesthesia using fentanyl $(20-50 \mu \mathrm{g} / \mathrm{kg} / \mathrm{h})$ and muscular blockade with pancuronium $(0.3 \mathrm{mg} / \mathrm{kg} / \mathrm{h})$. Oxygen was added to the respiratory circuit with the aim of achieving a peripheral arterial saturation of greater than $94 \%$. Invasive arterial blood pressure was measured through the side port of the arterial access in the carotid or femoral artery. Peripheral arterial saturation was monitored with a pulse-oximeter in a continuous fashion. Postprocedural buprenorphine $(0.01 \mathrm{mg} / \mathrm{kg}$ twice daily) was used for analgesia. Cefazolin $(25 \mathrm{mg} / \mathrm{kg})$ was used for antibiotic prophylaxis. 
Timeline of the experimental series

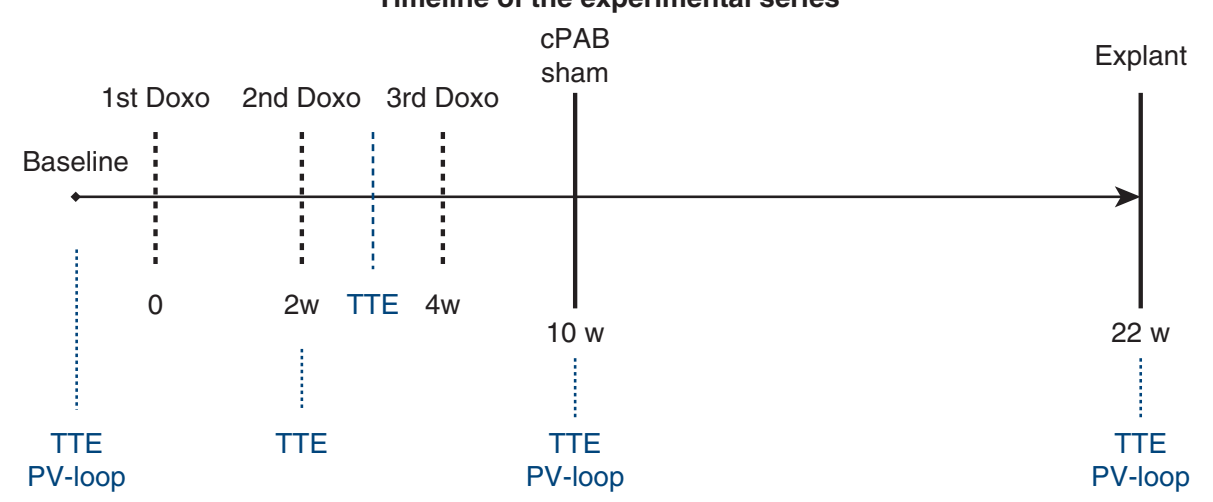

FIGURE 1. Timeline of the experimental series showing the timing of serial (3) intracoronary doxorubicin injections, sham/cPAB operation, and final operation for hemodynamic assessment and harvesting the hearts. The type and timing of the hemodynamic investigations are shown in blue font color. Doxo, Doxorubicin; $C P A B$, central pulmonary artery banding; TTE, transthoracic echocardiography; $P V$, pressure-volume.

\section{Coronary Angiography and Doxorubicin Application}

The timeline of the experiments and performed investigations is depicted in Figure 1. Up to 3 intracoronary injections of doxorubicin (each dose $0.75 \mathrm{mg} / \mathrm{kg}$ ) were administered every 2 to 6 weeks until echocardiographic signs of left ventricular functional impairment and spatial dilation were observed. After detection of left ventricular dysfunction and spatial dilation, surgical pulmonary banding was planned 3 to 4 weeks after the last doxorubicin injection.

For the 2 initial doxorubicin injections, a $5 \mathrm{~F}$ introducer was inserted into the left carotid artery. For the third injection, the left or right femoral artery was percutaneously cannulated with a $5 \mathrm{~F}$ introducer. After a $100 \mathrm{IE} / \mathrm{kg}$ heparin bolus was administered, a $5 \mathrm{~F}$ pigtail calibration catheter was advanced into the left ventricle under fluoroscopy, and a standard 1-plane cineventriculography at 25 frames per second was performed (Siemens, Arcadis Avantic, Germany). With lateral imaging, a 5F right Judkins catheter (Infiniti JR4, Cordis, Milpitas, Calif) was used to catheterize the left coronary ostium. Adequate placement of the catheter's tip was checked with small boluses of contrast media. A constant rate of intracoronary infusion of $0.75 \mathrm{mg} / \mathrm{kg}$ doxorubicinhydrochloride solution (Applichem GmbH, Darmstadt, Germany) diluted in $10 \mathrm{~mL}$ of saline was started with a syringe pump and maintained over 10 minutes. Animals were closely monitored in the immediate postoperative period for signs of arrhythmia or ventricular dysfunction.

\section{Hemodynamic Assessment}

Transthoracic echocardiography. Transthoracic conventional echocardiography was performed by cardiologists experienced in pediatric and adult echocardiography while the animals were under sedation (or endotracheal intubation if there was a planned heart catheterization). Echocardiography was performed at baseline, before each doxorubicin injection, 2 to 4 weeks after the last doxorubicin injection, before cPAB, and before the explantation procedure. A Phillips (Andover, Mass) CX

TABLE 1. Hemodynamic characteristics before pulmonary artery banding/sham operation in both groups

\begin{tabular}{|c|c|c|c|}
\hline & Sham & cPAB & $P$ value \\
\hline Echo LVEDd & $4.13 \pm 0.24$ & $4.27 \pm 0.19$ & .45 \\
\hline Echo LVESd & $3.11 \pm 0.25$ & $3.37 \pm 0.40$ & .49 \\
\hline Echo EDV (mL) & $75.83 \pm 10.22$ & $81.79 \pm 8.34$ & .46 \\
\hline Echo ESV (mL) & $38.74 \pm 7.32$ & $47.37 \pm 12.69$ & .46 \\
\hline Echo EF (\%) & $48.71 \pm 7.78$ & $43.14 \pm 10.50$ & .56 \\
\hline Heart rate $\left(\min ^{-1}\right)$ & $109.43 \pm 23.11$ & $143.00 \pm 31.79$ & .30 \\
\hline $\mathrm{ESP}(\mathrm{mm} \mathrm{Hg})$ & $103.86 \pm 21.47$ & $129.67 \pm 13.72$ & .11 \\
\hline $\mathrm{EDP}(\mathrm{mm} \mathrm{Hg})$ & $9.00 \pm 3.41$ & $14.00 \pm 3.00$ & .38 \\
\hline $\mathrm{SW}(\mathrm{mL} / \mathrm{mm} \mathrm{Hg})$ & $4867.29 \pm 2101.23$ & $5642.00 \pm 793.84$ & .47 \\
\hline $\mathrm{dp} / \mathrm{dt}_{\max }\left(\mathrm{mm} \mathrm{Hg} \times \mathrm{s}^{-1}\right)$ & $1257.14 \pm 777.12$ & $1827.67 \pm 932.18$ & .51 \\
\hline $\mathrm{dp} / \mathrm{dt}_{\min }\left(\mathrm{mm} \mathrm{Hg} \times \mathrm{s}^{-1}\right)$ & $-2008.86 \pm 964.39$ & $-2280.67 \pm 575.25$ & .65 \\
\hline tau (ms) & $63.00 \pm 69.42$ & $37.67 \pm 10.34$ & .42 \\
\hline Ees $(\mathrm{mm} \mathrm{Hg} / \mathrm{mL})$ & $1.10 \pm 0.38$ & $1.86 \pm 0.23 *$ & .02 \\
\hline Eed $(\mathrm{mm} \mathrm{Hg} / \mathrm{mL})$ & $0.18 \pm 0.06$ & $0.27 \pm 0.05$ & .15 \\
\hline
\end{tabular}

Bold values represent significance $(P<.05)$. Values are presented as mean \pm standard deviation. $c P A B$, Central pulmonary artery banding; $L V E D d$, left ventricular end diastolic diameter; $L V E S d$, left ventricular end systolic diameter; $E D V$, end-diastolic volume; $E S V$, end-systolic volume; $E F$, ejection fraction; $E S P$, end-systolic pressure; $E D P$, enddiastolic pressure; $S W$, stroke work; $d p / d t_{\max }$ maximal slope of systolic pressure increment; $d p / d t_{\min }$, diastolic pressure decrement; tau, left ventricular diastolic time constant; Ees, slope of end-systolic pressure volume relation; Eed, slope of end-diastolic pressure volume relation. 

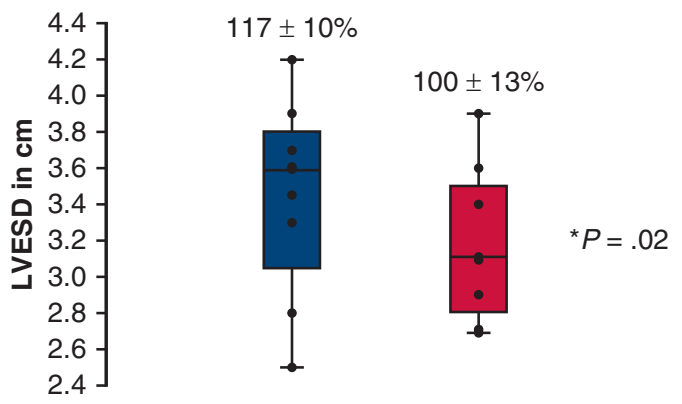

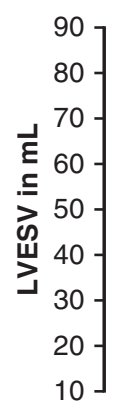

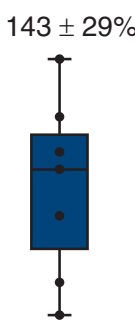

A

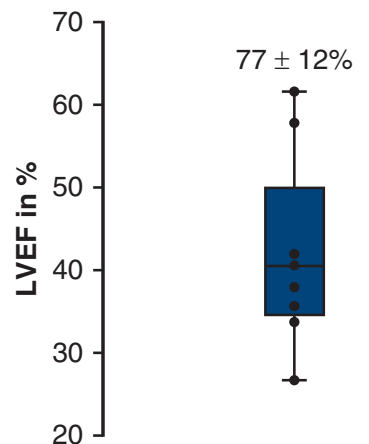

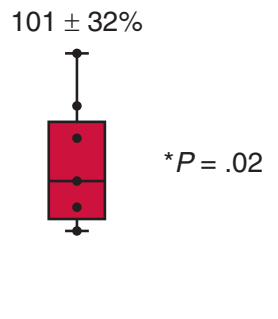

Sham $(n=8)$

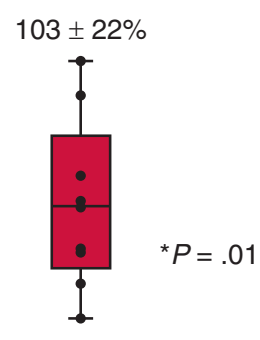

Sham $(n=8)$
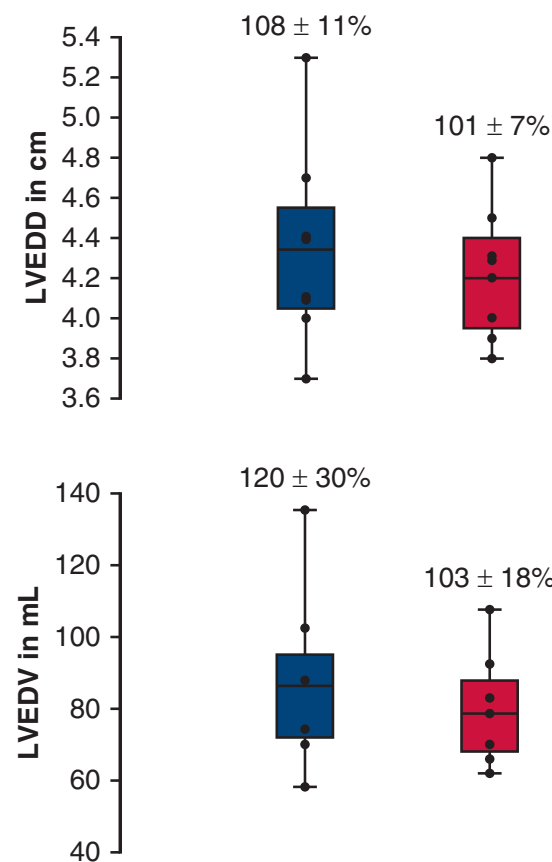

$P=.18$

$P=.09$
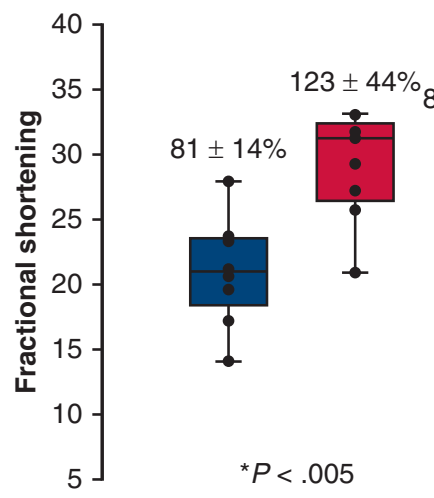

$119 \pm 44 \%$

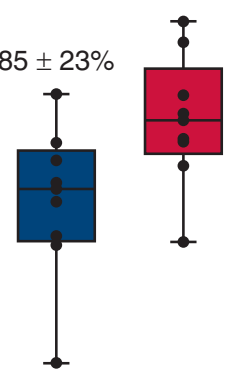

${ }^{\star} P=.016$

\section{B}

$\operatorname{cPAB}(n=9)$

FIGURE 2. A and B, Transthoracic echocardiographic measurements of the sham and cPAB groups before the explantation of the hearts at the end of the experimental series. The $X$-axis shows the different groups. The upper and lower borders of the boxes represent the upper and lower quartiles. The middle horizontal line is the median value. Each measurement is shown as a black dot, and the dots outside of the box and whiskers represent outliers. Percentages above the box plots show the relation of each parameter to the baseline level at before cPAB/sham operation $(100 \%)$. $*<<.05$. Blue color: sham group, green color: cPAB group. $L V E S d$, Left ventricular end-systolic diameter; $L V E D d$, left ventricular end-diastolic diameter; $L V E S V$, left ventricular end-systolic volume; $L V E D V$, left ventricular end-diastolic volume; $c P A B$, central pulmonary artery banding; $L V E F$, left ventricular ejection fraction.

50 System equipped with an S8-3 MHz transducer was used for continuous electrocardiogram monitoring of the animals. The animals were anesthetized with intravenous injections of $0.5 \mathrm{mg} / \mathrm{kg}$ midazolam and $10 \mathrm{mg} / \mathrm{kg}$ ketamine. Oxygen was supplied with a face mask. The animals were placed in ventral recumbency, and wool was clipped between the right fourth and seventh intercostal spaces. All transthoracic echocardiographic measurements included a mean of 3 consecutive beats.

Left ventricular dimension measurements were performed using 2dimensional-guided M-mode on the right parasternal ventricular short- axis view, according to the recommendations of the American Society of Echocardiography. Left ventricular end-systolic and end-diastolic diameters, left ventricular end-systolic and diastolic volumes, and left ventricular ejection fraction (LVEF) were measured, and left ventricular shortening fractions were then calculated in the short-axis and long-axis.

Pressure-volume loop measurements using conductance technique. Indices of left ventricular function using a conductance catheter technique were derived at 2 different time points: (1) before the 


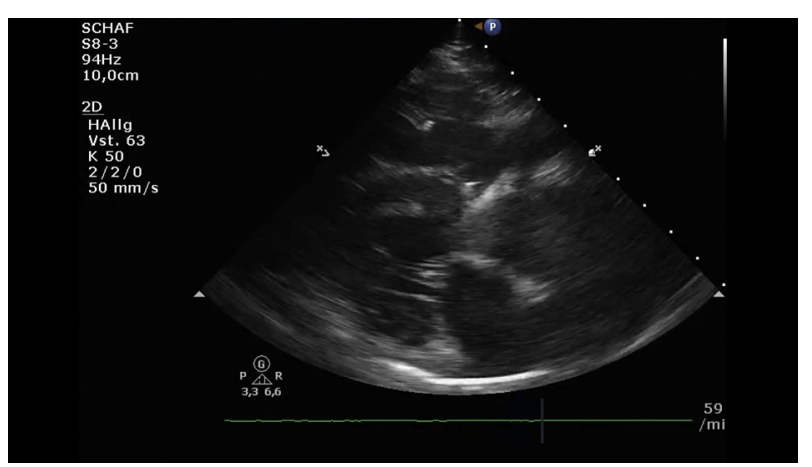

VIDEO 1. The transthoracic echocardiographic assessment of a representative animal from the CPAB group at 3 different time points is shown: (1) at baseline before the doxorubicin treatment; (2) after doxorubicin treatment before $\mathrm{cPAB}$; and (3) 3 months after cPAB. Video available at: https:// www.jtcvs.org/article/S0022-5223(19)30535-5/fulltext.

start of doxorubicin injection into the coronary arteries and (2) before the explantation of the hearts 3 months after the cPAB procedure. Assessment of left ventricular intrinsic myocardial function by analysis of pressurevolume (PV) loops was performed using cardiac function hardware (INCA, CD Leycom, Hengelo, The Netherlands) and software package version 3.8 (2008; CD Leycom). To acquire PV loops, a 4F conductance catheter with 12 electrodes and integrated manometer (41063-PN, CD Leycom) was placed in the ventricle via left carotid artery cannulation and the ascending aorta. A gradual reduction in ventricular volume (preload reduction) was induced by occlusion of the inferior cava vein using an $8 / 22$ Fogarty catheter (Edward Lifesciences $\mathrm{GmbH}$, Unterschleissheim, Germany) that was introduced through the right internal jugular vein. Recordings were made during at least 3 maneuvers in each subject. If ectopic beats occurred during the preload reduction, the recording was repeated to obtain at least 2 acceptable measurements. All measurements were performed during short periods of suspended ventilation at end expiration. Heart rate, end-diastolic pressure, end-systolic pressure, and maximal rate of pressure change (maximal slope of systolic pressure increment $\left[\mathrm{dp} / \mathrm{dt}_{\max }\right]$ ) were analyzed. Stroke work was calculated as the area enclosed by the PV loop. Indices of load-independent systolic and diastolic left ventricular function were obtained from PV loops recorded during preload reduction by the slope of the end-systolic and end-diastolic PV relation. The time constant of relaxation $(\tau)$, reflecting the early active relaxation process, was calculated as the time constant of the monoexponential pressure decay during isovolumetric relaxation. The isovolumetric period was defined as the period between the time point of maximal slope of diastolic pressure decrement $\left(\mathrm{dp} / \mathrm{dt}_{\min }\right)$ and the time point at which $\mathrm{dp} / \mathrm{dt}$ reached $10 \%$ of the $\mathrm{dp} / \mathrm{dt}_{\min }$ value.

Central pulmonary artery banding/sham procedure. After the documentation of a dilated cardiomyopathy of the left ventricle, surgical pulmonary artery banding (PAB) was performed in the $\mathrm{CPAB}$ group. This was achieved by banding of the pulmonary trunk with a 2$\mathrm{mm}$-wide Dacron band via a left anterior thoracotomy through the fifth or sixth intercostal space. The degree of the pulmonary stenosis was gradually adjusted by direct intraventricular measurements with a pressure tip catheter and an elevation of the systolic right ventricular maximal pressure of $50 \%$ to $60 \%$ above the baseline level. When the desired pressure level was reached, the 2 ends of the Dacron band were fixed using 2 stitches through both ends of the band with 5-0 Prolene. The band was fixed on the wall of the pulmonary trunk using single 5-0 Prolene stitches on each side to prevent inadvertent sliding of the band toward the pulmonary bifurcation. The sham animals received a left anterior thoracotomy through the fifth or sixth intercostal space with pericardial opening only.
Descriptions of the macroscopic organ inspection and microscopic investigation of inflammatory cell load and fibrosis are available in the Appendix E1.

\section{Statistics}

Data were analyzed for normal distribution using the KolmogorovSmirnov test. Pairwise comparison was performed using a $t$ test for normally distributed data and the Mann-Whitney $U$ test for not normally distributed data. Comparison between more than 2 groups was performed using 1-way analysis of variance testing followed by post hoc correction using Tukey's test. Statistically significant differences were indicated by single asterisks $(* P<.05)$.

\section{RESULTS \\ Survival}

Two animals were excluded from the study because of other illnesses before the cPAB. Mortality occurred in 8 of the 11 cases before the $\mathrm{CPAB}$. Eight animals died during the period between the start of the doxorubicin injections and the PAB procedure ( 3 animals during anesthesia application, 1 animal had malignant arrhythmia during cardiac catheterization, and 4 animals after anesthesia for the echocardiography and cardiac catheterization due to unknown postprocedural reasons in the barn).

There were 3 operative deaths during or after $\mathrm{CPAB}$ or sham operation (1 animal from the PAB group and 2 animals from the sham group; 1 due to surgical bleeding from the aorta, and 2 due to hemodynamic deterioration, 1 early and 1 two weeks after the operation, respectively). Nine animals in the $\mathrm{CPAB}$ group and 8 animals in the sham group with similar weights survived and were included in the final analyses.

\section{Myocardial Function and Dimensions}

Echocardiographic results. There were no differences in the echocardiographic measurements before the index operation of $\mathrm{CPAB}$ or sham operation between the 2 groups. These measurements were considered as the baseline level $(100 \%)$ (Table 1). The subsequent echocardiographic results were calculated as percentages of the baseline before the explantation of the hearts after a median follow-up of 3 months. The absolute values are presented in median and interquartile ranges in Figure 2, $A$ and $B$.

Before the explantation of the hearts, the cPAB group had better LVEF $(102.5 \% \pm 21.6 \%$ vs $76.7 \% \pm 11.7 \%$, $P=.0197)$, with similar left ventricular end-diastolic volumes $(101.2 \% \pm 7.4 \%$ vs $120.4 \% \pm 10.8 \%, P=.1774)$ and significantly smaller end-systolic diameters $(100.3 \% \pm 12.9 \%$ vs $116.5 \% \pm 9.6 \%, P=.021)$ compared with the sham animals before heart explantation. The left ventricular end-systolic volume $(101.4 \% \pm 31.6 \%$ vs $143.4 \% \pm 28.6 \%, P=.0214$ ) was lower in the $\mathrm{CPAB}$ group, and the left ventricular end-diastolic volume $(103.3 \% \pm 18 \%$ vs $120.4 \% \pm 29.5 \%, P=.092)$ was similar between the groups. There were significantly higher values for fractional shortening in the long axis 

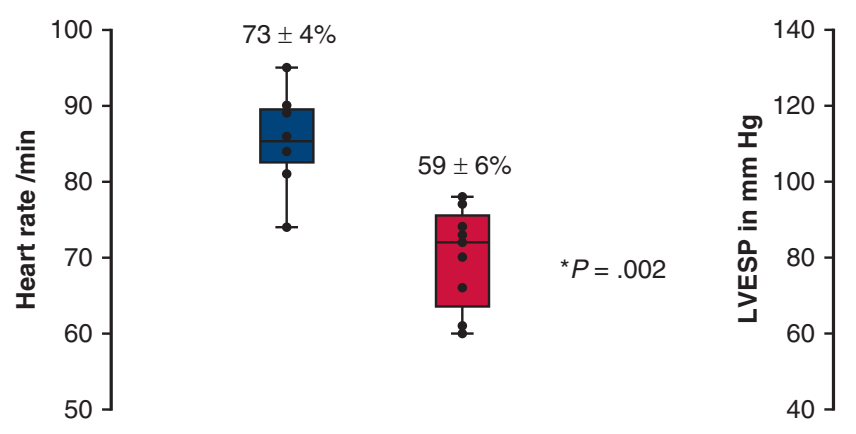

$100 \pm 15 \%$
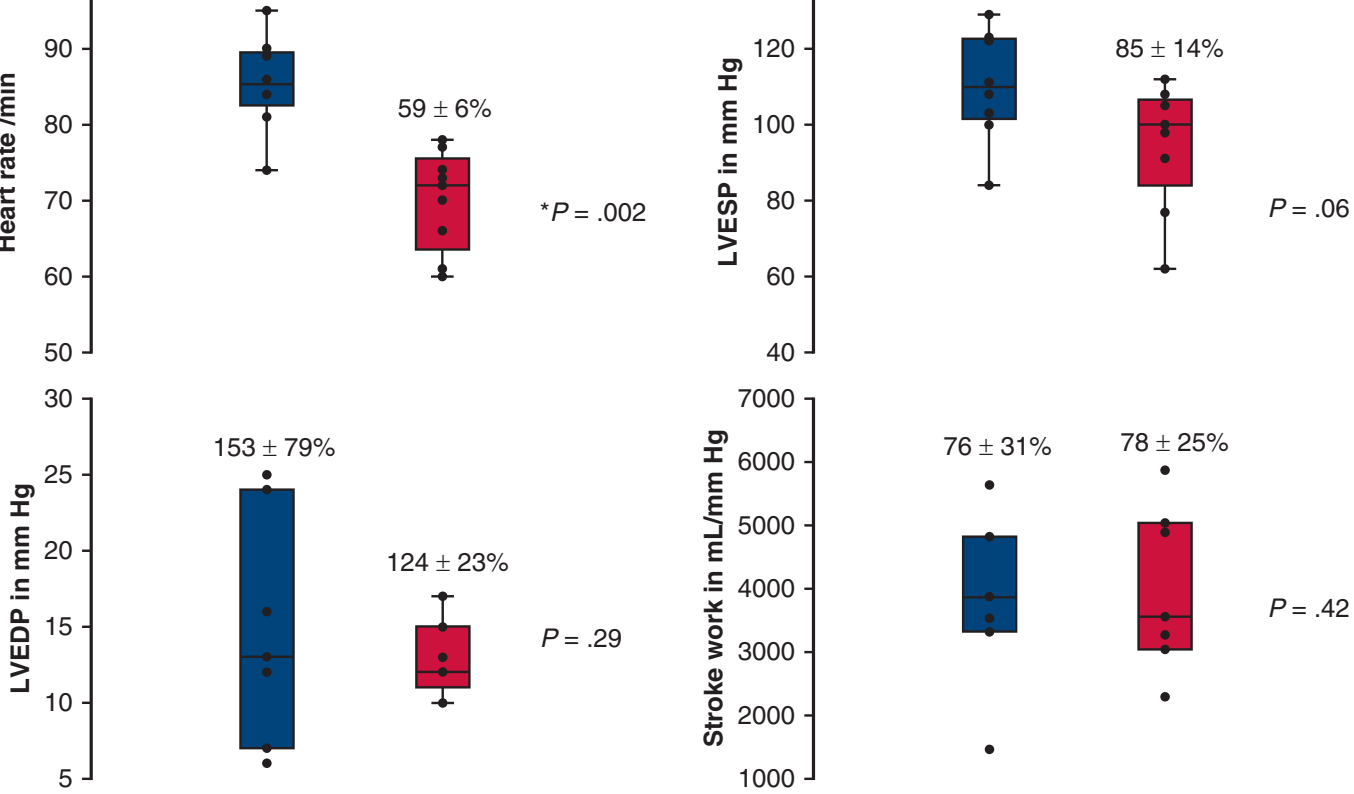

$78 \pm 25 \%$

A

Sham $(n=8)$

$\mathrm{cPAB}(\mathrm{n}=9)$

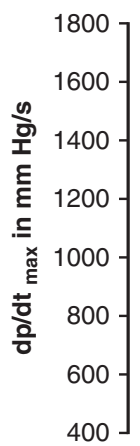
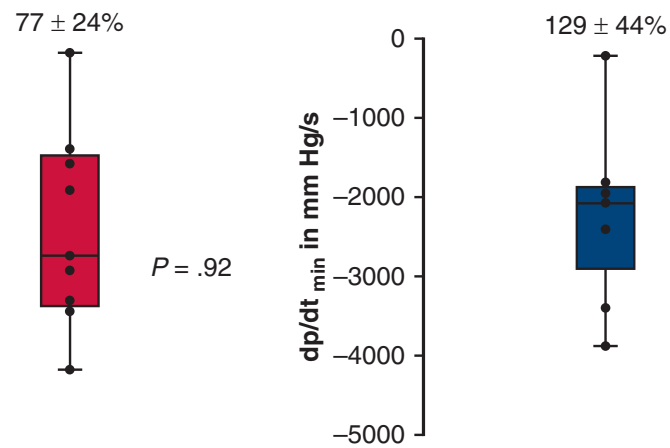

$91 \pm 25 \%$

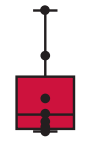

$P=.05$

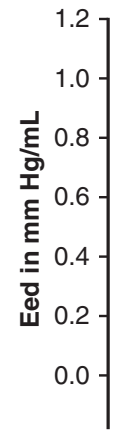

$194 \pm 153 \%$

B

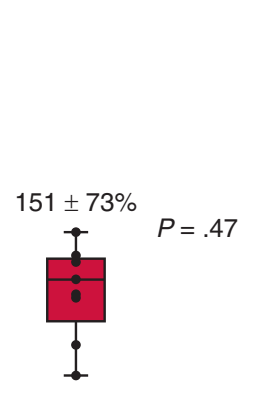

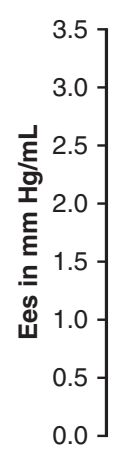

$141 \pm 76 \%$
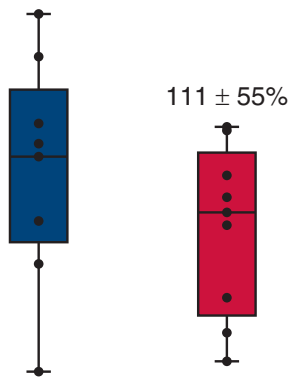

$P=.39$

Sham $(n=8)$

$\mathrm{cPAB}(n=9)$

FIGURE 3. A and B, Conductance catheter measurements with PV loop assessment of the sham and cPAB groups before the explantation of the hearts at the end of the experimental series. The $X$-axis shows the different groups. The upper and lower borders of the boxes represent the upper and lower quartiles. The middle horizontal line is the median value. Each measurement is shown as a black dot, and the dots outside of the box and whiskers represent outliers. Percentages above the box plots show the relation of each parameter to the baseline level at before cPAB/sham operation $(100 \%)$. ${ }^{*} P<.05$. Blue color: sham group, green color: $\mathrm{CPAB}$ group. $L V E D P$, Left ventricular end-diastolic pressure; $L V E S P$, left ventricular end-systolic pressure; $c P A B$, central pulmonary artery banding; $d p / d t_{\max }$, maximal slope of systolic pressure increment; $d p / d t_{\min }$, diastolic pressure decrement; Eed, slope of end-diastolic pressure volume relation; Ees, slope of end-systolic pressure volume relation. 

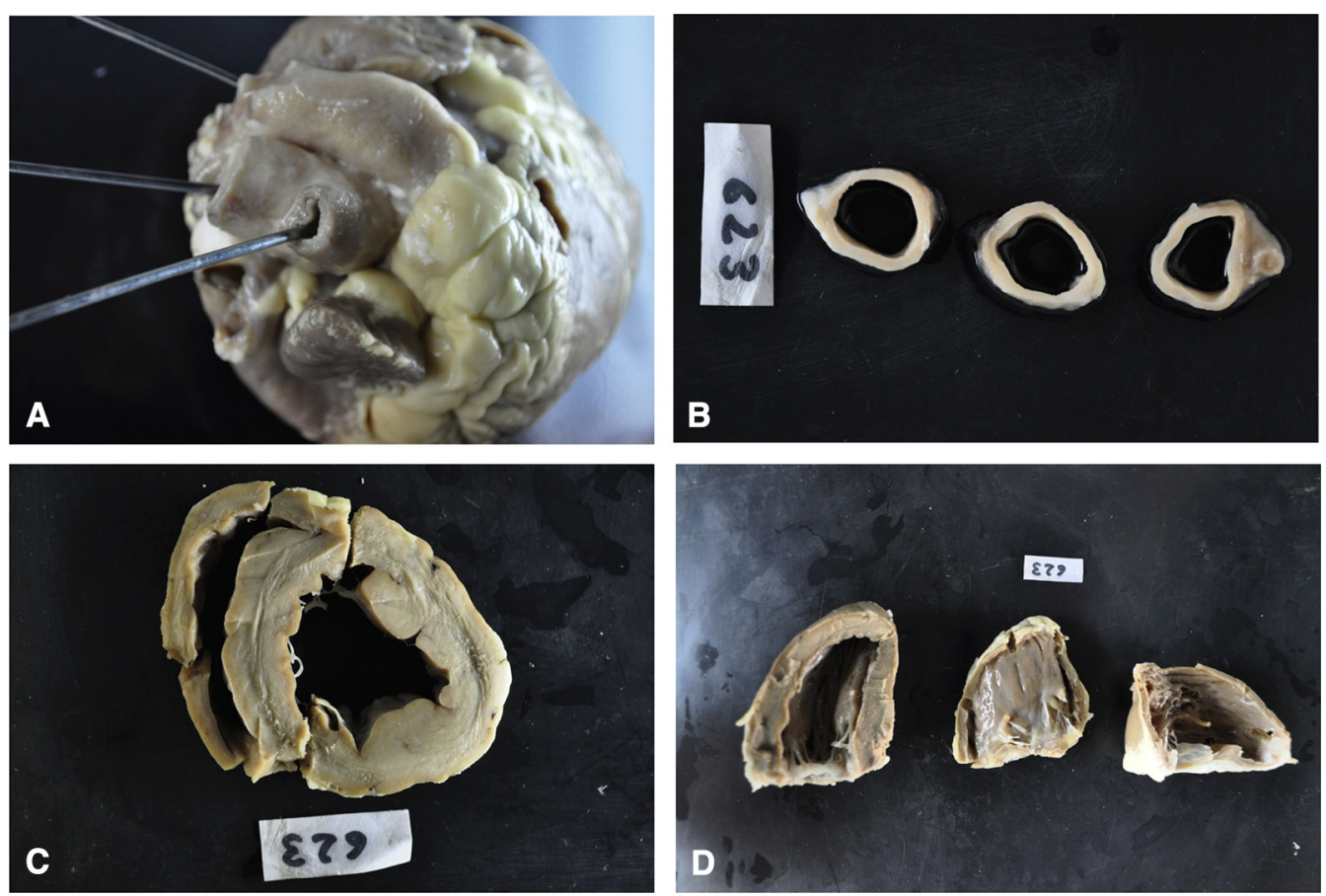

FIGURE 4. Macroscopic appearance of the heart of 1 representative animal from the sham group. A, Entire heart showing the main pulmonary artery and the aorta (probes). B, Sections at 3 different levels of the main pulmonary artery. C, Midventricular cross-section of the left and right ventricular cavities; note the septal shift to the right. D, Left ventricular cavity, septal left ventricular surface, and right ventricular cavity (longitudinal section) from left to right.

$(118.5 \% \pm 21.5 \%$ vs $85.2 \% \pm 22.8 \%, P=.0164)$ and short axis $(122.5 \% \pm 18 \%$ vs $80.9 \% \pm 13.6 \%$, $P=.0005$ ) in the cPAB group (Figure 2, $A$ and $B$ ). The echocardiographic findings of 1 representative animal from the $\mathrm{cPAB}$ group throughout the experiments are presented in Video 1.

\section{Pressure-volume loop measurements}

There were no differences between the groups in the baseline PV loop measurements. During the PV loop assessments before explantation, we noted a lower heart rate in the $\mathrm{CPAB}$ group $(58.7 \pm 5.5$ beats $/ \mathrm{min}$ vs $72.6 \pm 4.2$ beats $/ \mathrm{min}$, $P=.002)$. No significant differences were seen for the parameters of systolic function, such as $\mathrm{dp} / \mathrm{dt}_{\max }$, stroke work, and end-systolic elastance and left ventricular end-systolic pressure $(84.9 \pm 14.3 \mathrm{~mm} \mathrm{Hg}$ vs $100.4 \pm 14.7 \mathrm{~mm} \mathrm{Hg}$, $P=.0636)$. With regard to diastolic functional properties, the $\mathrm{dp} / \mathrm{dt}_{\min }$ was similar in both groups $(91.2 \pm 24.9 \mathrm{~mm}$ $\mathrm{Hg} / \mathrm{s}$ vs $128.8 \pm 43.5 \mathrm{~mm} \mathrm{Hg} / \mathrm{s}, P=.0591)$. Likewise, enddiastolic elastance and diastolic time constant end-systolic and end-diastolic pressures were similar in the $\mathrm{CPAB}$ and sham groups (Figure 3, $A$ and $B$, and Figure E1).

\section{Macroscopy}

Macroscopic examinations confirmed the narrowing of the pulmonary artery using the $\mathrm{CPAB}$ in comparison with the sham operation. The right ventricular and right atrial wall thicknesses were significantly more pronounced in the cPAB group $(P=.04$ and $P=.0042$, respectively). Significant differences were also noticed in the dimensions of the main pulmonary artery due to the cPAB, with a smaller dimension observed on the banding site and a larger diameter proximal to the banding in comparison with the sham group $(P<.05$ and $P<.01$, respectively). The macroscopic appearance of subendocardial fibrotic changes was noted and documented in most of the animals in the region of the papillary muscles and interventricular septum. In consideration of this article's length, only significant macroscopic measurements are reported (Figures 4 and 5; Tables E1-E4).

\section{Microscopic examinations}

The area of fibrosis and the inflammatory cell count in both the $\mathrm{CPAB}$ and sham groups did not differ significantly in the Picrosirius red and hematoxylin-eosin staining, respectively. There was a tendency for higher values of fibrosis area and inflammatory cell count in the experimental cohort than in hearts from healthy controls (Figure 6, $A$ and $B$ ).

\section{DISCUSSION}

CPAB was first implemented to restrict excessive pulmonary blood flow in cases with left-to-right intracardiac shunting. ${ }^{13}$ Over the years, several other applications were introduced. ${ }^{14}$ 

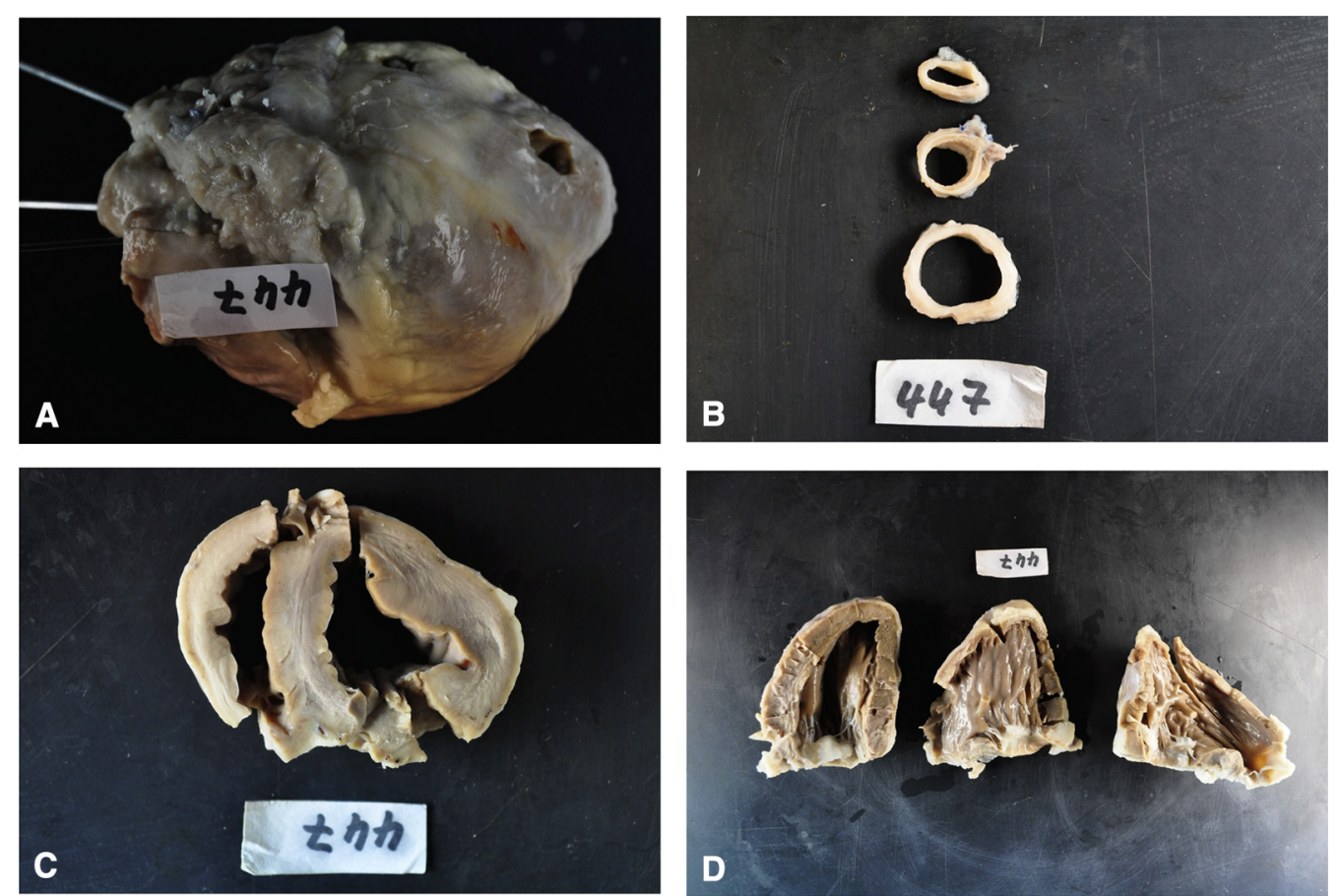

FIGURE 5. Macroscopic appearance of the heart of 1 representative animal from the cPAB group. A, Entire heart showing the main pulmonary artery (note the banding) and the aorta (probes). B, Sections at 3 different levels of the main pulmonary artery (note the narrowing at the banding site). C, Midventricular cross-section of the left and right ventricular cavities (note the right ventricular hypertrophy). D, Left ventricular cavity, septal left ventricular surface, and right ventricular cavity (longitudinal section) from left to right.

In 2007, the Giessen group from Germany reported the first application of CPAB for the treatment of left ventricular cardiomyopathy in a pediatric patient. ${ }^{9}$ Further data have been introduced from the Giessen group as well as others over the past several years. ${ }^{10,15}$ A summary of the worldwide experience of $\mathrm{CPAB}$ for the treatment of left ventricular cardiomyopathy was most recently reported by Schranz and colleagues. ${ }^{11}$

This is the first experimental series worldwide to use surgical $\mathrm{CPAB}$ treat toxic dilated cardiomyopathy in a sheep model. Other groups have published the method of intracoronary doxorubicin infusion with successful creation of a dilated left ventricle with reduced function. ${ }^{12}$ The standard technical approach of the Giessen group was replicated in this experimental work for surgical $\mathrm{CPAB}$ with direct intracardiac pressure measurements for pulmonary band adjustment. In our series, the intracoronary application of doxorubicin led to left ventricular dilation and reduced left ventricular function with a reduction in LVEF and fractional shortening on transthoracic echocardiography.

After surgical cPAB, we observed significantly lower left ventricular end-systolic diameters and volumes before explantation of the hearts. The $\mathrm{cPAB}$ group had greater LVEF and short- and long-axis fractional shortening 3 months after the banding procedure. The findings should be accepted in the context of a lower heart rate in the cPAB group before the explantation of the hearts $(58.7 \pm 5.5$ beats $/ \mathrm{min}$ vs $72.6 \pm 4.2$ beats $/ \mathrm{min}, P=.002$ ). The observed lower heart rate can be interpreted as a sign of less pronounced heart failure in the cPAB group, because a higher heart rate is known to be associated with a worse prognosis in the context of adult chronic heart failure. The lower heart rate may have affected our echocardiographic findings; however, no animal had tachycardia per the definition, so the effect of the different heart rates between the groups on the echocardiographic results is expected to be limited. Conductance catheter measurements with the assessment of PV loops failed to demonstrate any significant differences between the groups, but there was a trend to a lower end-systolic pressure in the left ventricle of the cPAB group $(P=.06)$. The reasons for this discrepant finding may be that we had relatively significant outliers in the PV loop measurements in both groups, the duration of 3 months may not be long enough to see a significant improvement in the intrinsic contractile properties of the ventricle, and the cardiomyopathy observed in this study had a toxic nature with a different cause than dilated cardiomyopathy in childhood.

The outcomes of infants and young children who undergo cardiac transplantation for dilated cardiomyopathy worsen with the severity of left ventricular dilation. ${ }^{16}$ In the setting of pressure overload of the right heart, one of the potential mechanisms of reduced left ventricular dimensions and 

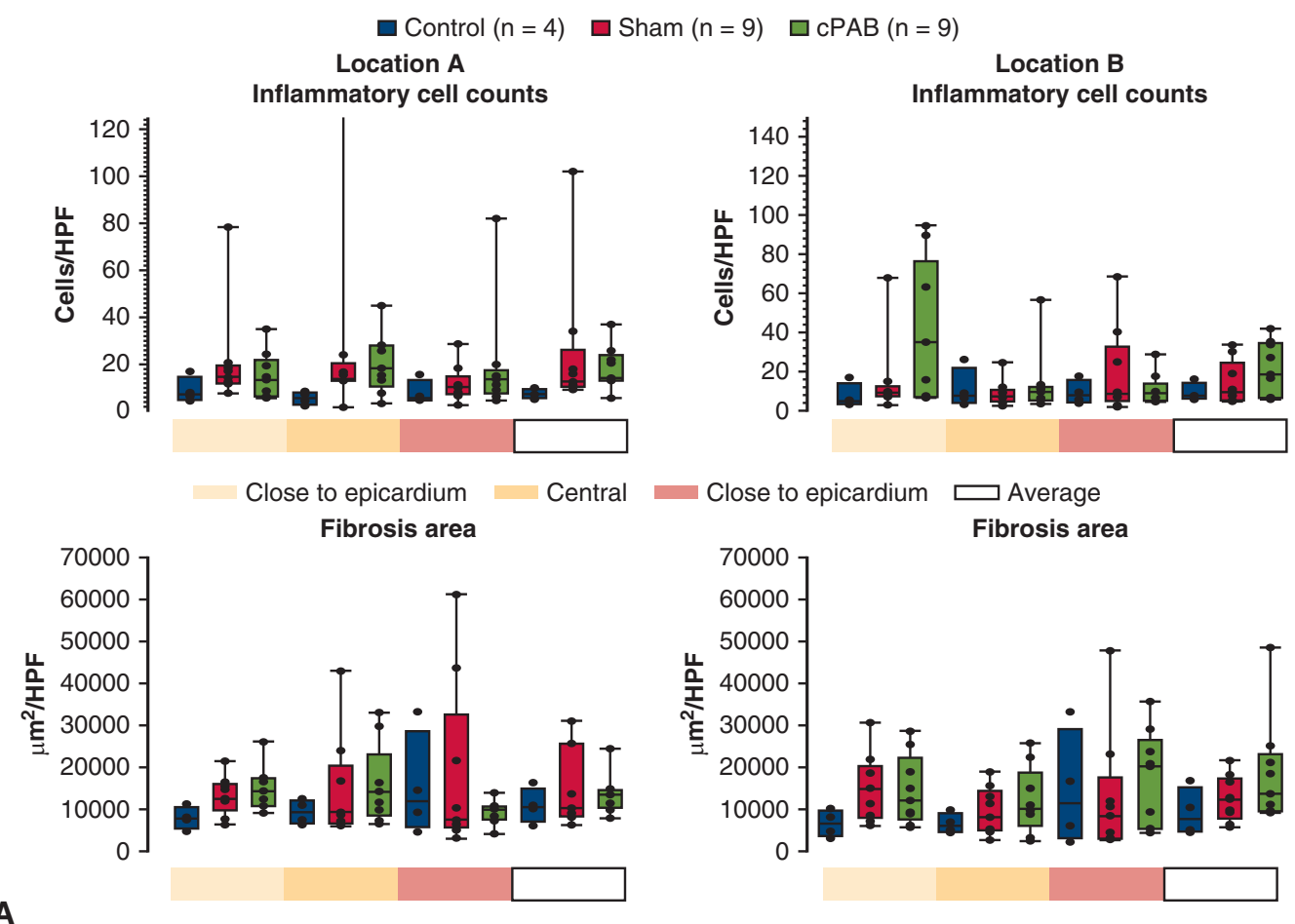

A
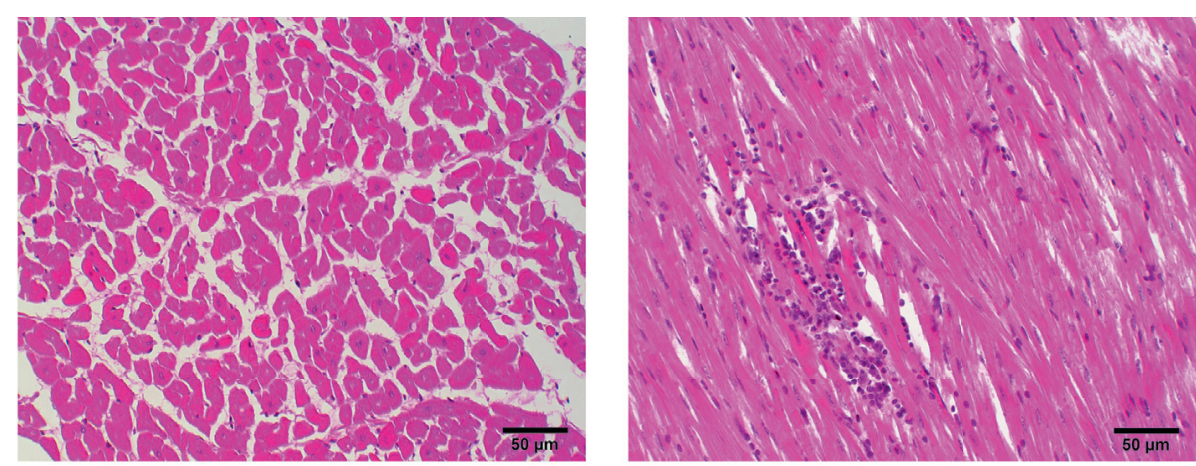

H.E. staining, 20x

H.E. staining, 20x
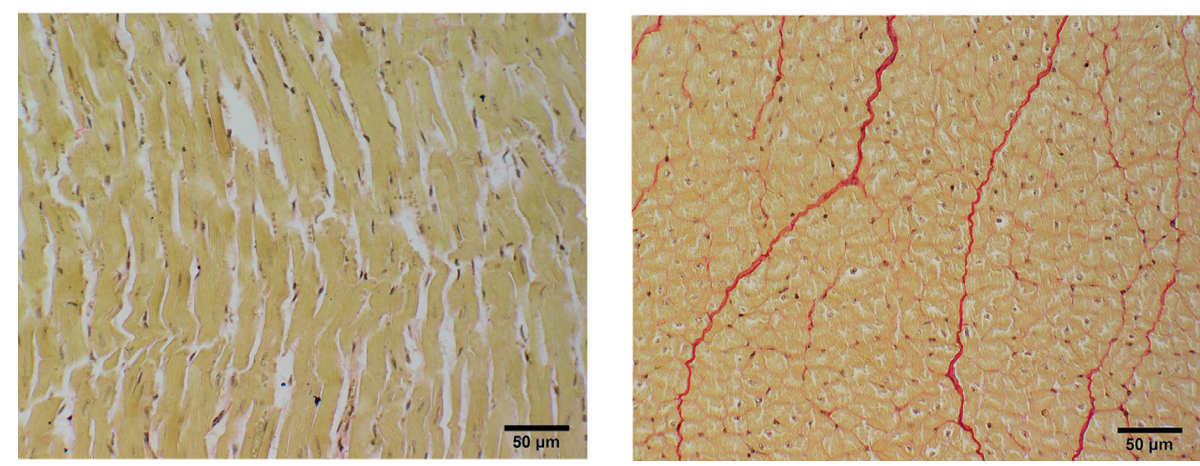

B

Picrosiriusred staining, 20x

Picrosiriusred staining, 20x

FIGURE 6. A, Inflammatory cell count (top) and fibrosis area (bottom) in the healthy control animals (yellow), sham group (blue), and cPAB (green) group in locations A and B separated in 4 different levels of the myocardium. Light yellow: close to epicardium, yellow: central myocardium, pink: close to endocardium, white: average value of all 3 levels. The $X$-axis shows the different groups. The upper and lower borders of the boxes represent the upper and lower quartiles. The middle horizontal line is the median value. Each measurement is shown as a black dot, and the dots outside of the box and whiskers represent outliers. B, Picrosirius and hematoxylin-eosin staining in healthy animals (left) and cPAB group (right). Inflammatory changes and increased fibrosis were seen in both experimental groups (sham and $\mathrm{cPAB}$ ), but more prominently than in the healthy controls. HPF, High-power field. 


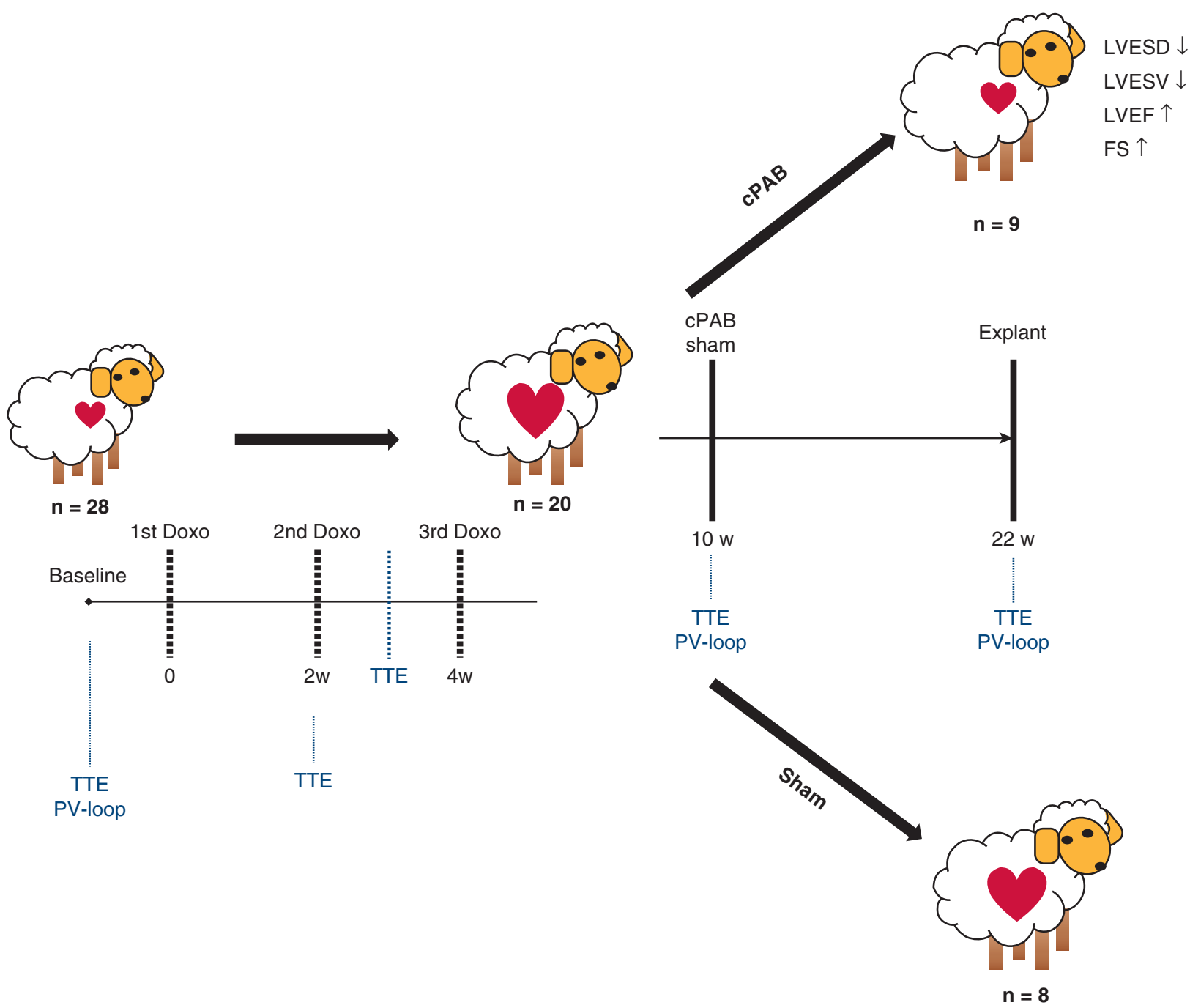

FIGURE 7. Four-month-old sheep $(\mathrm{n}=28)$ were treated with intermittent intracoronary injections of doxorubicin $(0.75 \mathrm{mg} / \mathrm{kg} / \mathrm{dose}) \mathrm{into}$ the left main coronary artery until functional impairment and spatial dilation in the transthoracic echocardiography. Animals underwent a sham/cPAB operation 3 months after the sham/cPAB procedure with hemodynamic assessment using transthoracic echocardiography and PV loop assessment before harvesting the hearts. Animals that underwent cPAB showed significant improvement in left ventricular dimensions and volume (LVESD, LVESV) and left ventricular function (FS, LVEF) in comparison with the sham group before harvesting the hearts for histologic investigations. LVESD, Left ventricular end-systolic diameter; $L V E S V$, left ventricular end-systolic volume; $L V E F$, left ventricular ejection fraction; $F S$, fractional shortening; Doxo, doxorubicin; $c P A B$, central pulmonary artery banding; TTE, transthoracic echocardiography; $P V$, pressure-volume.

improved left ventricular function might be the leftward shift of the interventricular septum. This mechanism and its mechanical effects on the left ventricular function have been described in patients with pulmonary hypertension. ${ }^{17}$ We observed a septal shift in the cPAB group in the echocardiographic evaluations, but the extent was not quantified to serve as a reliable marker for the improved left ventricular dimensions in our experimental model. The functional effects of septal shift on the clinical application of cPAB for left ventricular cardiomyopathy have been shown. ${ }^{18}$ Furthermore, the septal shift may change the shape of the left ventricle and reduce the amount of mitral regurgitation, with the result of optimizing the volume overload of the left ventricle. The favorable change in the Frank-Starling curve and the reduction in left ventricular preload and enddiastolic/end-systolic filling pressures may enable a more favorable hemodynamic state. An enhanced right ventricular function has been shown to contribute to left ventricular function in the setting of cardiac resynchronization therapy in adults. ${ }^{19}$ The reduced preload conditions for the left ventricle could not be clearly demonstrated in our experimental series because the end-diastolic and end-systolic pressures were similar between the groups in the conductance catheter measurements $(P=.29$ and .06 , 
respectively). In contrast, in the $\mathrm{CPAB}$ group, the left ventricular end-systolic diameter and volume were significantly lower in comparison with the sham group without cPAB. Significantly higher LVEF and fractional shortening were also noted in the cPAB group.

Another potential mechanism that is proposed for the long-term improvement of left ventricular function by CPAB is the considerably higher regenerative capacity of the young heart. The regenerative capacity of the heart decreases with age, and strategies that rely on the regenerative potential of the young myocardium are promising. ${ }^{20} \mathrm{~A}$ stimulation of resident intracardiac stem cells and stem cell recruitment from the periphery may contribute to the improvement of left ventricular function. ${ }^{21,22}$ In our study, we did not detect any significant differences in the amount of fibrosis or inflammation within the CPAB and sham groups. This finding reveals similar effects of doxorubicin treatment on the left ventricular myocardium in both experimental groups.

We have observed a significant hypertrophy of the right ventricle in the macroscopic examinations as expected after effective PAB. Existing data describe a biological crosstalk between the right and left ventricles that may lead to the improvement of left ventricular function. ${ }^{23,24}$ Whether the Anrep effect, which leads to physiologically enhanced right ventricular contractility ${ }^{25}$ when the afterload increases following the Frank-Starling law, conveys its effect to the left side of the heart through a biological interaction (ie, the change in biventricular gene expression) remains speculative but possible. ${ }^{26}$

\section{Study Limitations}

Doxorubicin-induced cardiomyopathy leads to similar histologic changes as in dilated cardiomyopathy with degeneration of cardiomyocytes, vasculopathy, inflammatory infiltrates, and replacement fibrosis. However, the experimental animal model cannot fully simulate the clinical situation of a dilated cardiomyopathy in early childhood.

However, an in-depth analysis (ie, with immunohistochemistry) was not used; therefore, those subtle changes may not have been detected. This aspect is a significant limitation of our study that might have shown the effects of the $\mathrm{PAB}$ on the cellular level.

Adjunct medical heart failure treatment was not performed in this study, so we have likely not used the full potential of $\mathrm{CPAB}$ in these experiments. In the clinical setting, patients who undergo $\mathrm{CPAB}$ for left ventricular cardiomyopathy receive advanced intensive medical heart failure treatment after the procedure. ${ }^{27}$ The Giessen group reported that their advanced heart failure therapy may aid in the recovery and reverse remodeling of the left heart. ${ }^{28}$ In our experimental setting, cardiomyopathy was caused by a toxic cytostatic agent and is not comparable to the etiology of left ventricular cardiomyopathy in the clinical setting and experience. Furthermore, none of the animals received medical heart failure treatment postoperatively, so any potential beneficial effect is missing in this experimental series.

Right ventricular function and mitral regurgitation were not quantitated in our study; however, qualitative echocardiography did not reveal severe right ventricular dysfunction or mitral regurgitation in any of the animals. The heart rate in the CPAB group was lower before heart explantation. No animal was in a tachycardia state; nonetheless, our echocardiographic results, which may have been influenced by heart rate, should be accepted with caution.

\section{CONCLUSIONS}

cPAB in the experimental setting of toxic cardiomyopathy seems to improve only the echocardiographic left ventricular function and reduces left ventricular dimensions in the medium-term follow-up without an effect on the PV loop assessments (Figure 7). Whether the indication for a $\mathrm{CPAB}$ can be extended to further etiologies that lead to left ventricular functional compromise with spatial dilation and to older patients with left ventricular failure can be answered only with further detailed experimental work that can elucidate the underlying molecular mechanisms of this novel strategy. In addition, clinical application of this simple surgical technique, with low postoperative risk, in prospective, randomized multicenter studies for different etiologies may enable further understanding of its role in the treatment of dilated left ventricular cardiomyopathy.

\section{Webcast}

You can watch a Webcast of this AATS meeting presentation by going to: https://aats.blob.core.windows.net/media/ 18May01/28DE\%201.Cardiac\%20Surgery\%20Forum/ S74_6_webcast_080258710.mp4.

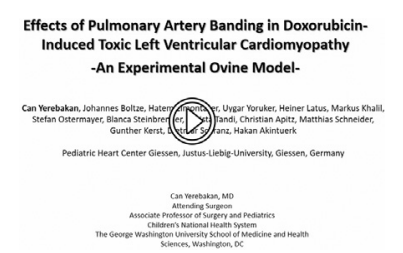

\section{Conflict of Interest Statement}

Authors have nothing to disclose with regard to commercial support.

The authors thank Dr Christa Tandi and Dr Alf Theisen from the J. W. Goethe University Frankfurt, Germany, for the veterinary support during the experimental series, and Katherine Klein for language editing of the manuscript. 


\section{References}

1. Lipshultz SE, Sleeper LA, Towbin JA, Lowe AM, Orav EJ, Cox GF, et al. The incidence of pediatric cardiomyopathy in two regions of the United States. $N$ Engl J Med. 2003;348:1647-55.

2. Nugent AW, Daubeney PEF, Chondros P, Carlin JB, Cheung M, Wilkinson LC, et al. The epidemiology of childhood cardiomyopathy in Australia. $N$ Engl J Med. 2003:348:1639-46.

3. Towbin JA, Lowe AM, Colan SD, Sleeper LA, Orav EJ, Clunie S, et al. Incidence, causes, and outcomes of dilated cardiomyopathy in children. JAMA. 2006;296: 1867-76.

4. Daubeney PEF, Nugent AW, Chondros P, Carlin JB, Colan SD, Cheung M, et al. Clinical features and outcomes of childhood dilated cardiomyopathy: results from a national population-based study. Circulation. 2006;114:2671-8.

5. Hsu DT, Canter CE. Dilated cardiomyopathy and heart failure in children. Heart Fail Clin. 2010;6:415-32.

6. Meyer P, Filippatos GS, Ahmed MI, Iskandrian AE, Bittner V, Perry GJ, et al. Effects of right ventricular ejection fraction on outcomes in chronic systolic heart failure. Circulation. 2010;121:252-8.

7. Schweiger M, Lorts A, Conway J. Mechanical circulatory support challenges in pediatric and (adult) congenital heart disease. Curr Opin Organ Transplant. March 20, 2018 [Epub ahead of print].

8. Das BB, Pruitt E, Molina K, Ravekes W, Auerbach S, Savage A, et al. The impact of flow PRA on outcome in pediatric heart recipients in modern era: an analysis of the pediatric heart transplant study database. Pediatr Transplant. 2018;22(1).

9. Schranz D, Veldman A, Bartram U, Michel-Behnke I, Bauer J, Akintürk H. Pulmonary artery banding for idiopathic dilative cardiomyopathy: a novel therapeutic strategy using an old surgical procedure. J Thorac Cardiovasc Surg. 2007;134: 796-7.

10. Schranz D, Rupp S, Müller M, Schmidt D, Bauer A, Valeske K, et al. Pulmonary artery banding in infants and young children with left ventricular dilated cardiomyopathy: a novel therapeutic strategy before heart transplantation. J Heart Lung Transplant. 2013;32:475-81.

11. Schranz D, Akintuerk H, Bailey L. Pulmonary artery banding for functional regeneration of end-stage dilated cardiomyopathy in young children: world network report. Circulation. 2018;137.

12. Borenstein N, Bruneval P, Behr L, Laborde F, Montarras D, Daurès JP, et al. An ovine model of chronic heart failure: echocardiographic and tissue Doppler imaging characterization. J Card Surg. 2006;21:50-6.

13. Muller WH Jr, Danimann JF Jr. The treatment of certain congenital malformations of the heart by the creation of pulmonic stenosis to reduce pulmonary hypertension and excessive pulmonary blood flow; a preliminary report. Surg Gynecol Obstet. 1952;95:213-9.

14. Winlaw DS, McGuirk SP, Balmer C, Langley SM, Griselli M, Stümper O, et al. Intention-to-treat analysis of pulmonary artery banding in conditions with a morphological right ventricle in the systemic circulation with a view to anatomic biventricular repair. Circulation. 2005;111:405-11.

15. Mets G, Panzer J, De Wolf D, Bove T. An alternative strategy for bridge-totransplant/recovery in small children with dilated cardiomyopathy. Pediatr Cardiol. 2017;38:902-8.

16. Alvarez JA, Orav EJ, Wilkinson JD, Fleming LE, Lee DJ, Sleeper LA, et al. Competing risks for death and cardiac transplantation in children with dilated cardiomyopathy: results from the pediatric cardiomyopathy registry. Circulation. 2011;124:814-23.

17. Dellegrottaglie S, Sanz J, Poon M, Viles-Gonzalez JF, Sulica R, Goyenechea M, et al. Pulmonary hypertension: accuracy of detection with left ventricular. Radiology. 2007;243:63-9.

18. Latus H, Hachmann P, Gummel K, Recla S, Voges I, Mueller M, et al. Biventricular response to pulmonary artery banding in children with dilated cardiomyopathy. J Heart Lung Transplant. 2016;35:934-8.

19. Lumens J, Ploux S, Strik M, Gorcsan J III, Cochet H, Derval N, et al. Comparative electromechanical and hemodynamic effects of left ventricular and biventricular pacing in dyssynchronous heart failure: electrical resynchronization versus left-right ventricular interaction. J Am Coll Cardiol. 2013;62:2395-403.

20. Bergmann O, Bhardwaj RD, Bernard S, Zdunek S, Barnabé-Heider F, Walsh S, et al. Evidence for cardiomyocyte renewal in humans. Science. 2009;324:98-102.

21. Smart N, Bollini S, Dubé KN, Vieira JM, Zhou B, Davidson S, et al. De novo cardiomyocytes from within the activated adult heart after injury. Nature. 2011;474:640-4.

22. Stellos K, Bigalke B, Langer H, Geisler T, Schad A, Kögel A, et al. Expression of stromal-cell-derived factor-1 on circulating platelets is increased in patients with acute coronary syndrome and correlates with the number of CD34+progenitor cells. Eur Heart J. 2009;30:584-93.
23. Roncon-Albuquerque R Jr, Vasconcelos M, Lourenco AP, Brandão-Nogueira A Teles A, Henriques-Coelho T, et al. Acute changes of biventricular gene expression in volume and right ventricular pressure overload. Life Sci. 2006;78: 2633-42.

24. Apitz C, Honjo O, Humpl T, Li J, Assad RS, Cho MY, et al. Biventricular structural and functional responses to aortic constriction in a rabbit model of chronic right ventricular pressure overload. J Thorac Cardiovasc Surg. 2012;144: 1494-501.

25. Yerebakan C, Klopsch C, Prietz S, Boltze J, Vollmar B, Liebold A, et al. Pressurevolume loops: feasible for the evaluation of right ventricular function in an experimental model of acute pulmonary regurgitation? Interact Cardiovasc Thorac Surg. 2009;9:163-8.

26. Yerebakan C, Klopsch C, Niefeldt S, Zeisig V, Vollmar B, Liebold A, et al. Acute and chronic response of the right ventricle to surgically induced pressure and volume overload - an analysis of pressure-volume relations. Interact Cardiovasc Thorac Surg. 2010;10:519-25.

27. Rupp S, Apitz C, Tholen L, Latus H, Ostermayer SH, Schmidt D, et al. Upgraded heart failure therapy leads to an improved outcome of dilated cardiomyopathy in infants and toddlers. Cardiol Young. 2015;25:1300-5.

28. Recla S, Steinbrenner B, Schranz D. Medical therapy in dilated cardiomyopathy and pulmonary arterial banding in children. J Heart Lung Transplant. 2013;32: 1045-6.

Key Words: cardiomyopathy, heart failure, pulmonary artery banding, left ventricle

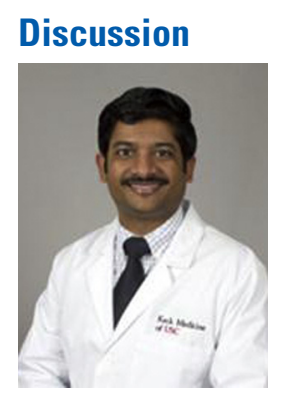

Dr Ram Kumar Subramanyan (Los Angeles, Calif). Yerebakan and colleagues provide valuable animal model data on the benefit of cPAB after doxorubicin-induced cardiac dysfunction. The concept is of significance to the scientific community, today's presentation was excellent, and the manuscript you sent me is absolutely well written. Congratulations on a job well done.

Dilated cardiomyopathy is a vexing problem in children and has limited surgical options. When there is a correctible congenital anatomic defect, the outcome can be impacted by surgically addressing the lesion. In the absence of a correctible anatomic lesion, options are limited to medical or device support and ultimately transplantation. Devising and testing novel surgical approaches to improve these outcomes are of intrinsic scientific merit, and this article is a laudable step in that direction.

Cam, I have a few questions for you. You rightly point out in your article the lack of a quality animal model of dilated cardiomyopathy. Your study was carried out in a doxorubicin-induced cardiac dysfunction model. Could you tell us why you believe this is a good surrogate for clinical dilated cardiomyopathy? In particular, have you done studies to show that there is thinning of the ventricular wall or magnetic resonance imaging to suggest that this mimics clinical dilated cardiomyopathy or develop mitral regurgitation, for example? 


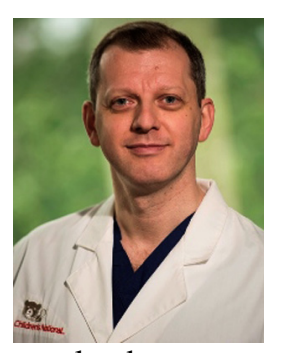

Dr Can Yerebakan (Washington, DC). I have been working for the last 10 years with animal models in Germany and looked for a valid model for dilated cardiomyopathy that is similar to the clinical scenario of patients we see in the neonatal phase. I then excluded all models with volume overload, pressure overload, which would not give us the features of a dilated cardiomyopathy in a short time period. Therefore, I searched for another model, and this is a toxic model, which actually disturbs the myocardial integrity similar to an acute myocarditis or idiopathic cardiomyopathy in childhood.

Borenstein and colleagues from France used this model and were able to show that doxorubicin leads to a dilated cardiomyopathy in a short time period of many weeks, and therefore I thought this model was the best one to choose from.

To your second question, we were able to show dilation of the left ventricular chamber. Unfortunately, these figures are not in the article but I will add them, but we showed spatial dilation and functional myocardial impairment. There was no myocardial thinning, so the measurements of the left ventricular wall in comparison with sham animals were not different. We were able to show right ventricular hypertrophy and left ventricular fibrosis, but no wall thinning. This is the answer for your second question.

Dr Subramanyan. In your discussion, you speculate on what the mechanisms of the observed benefit could be. The big advantage of an animal model is being able to ask mechanistic questions. So, do you have an idea or have you experimentally tested any of these hypotheses in your model? In particular, if I read your data correctly, the predominant benefit of PAB, in my opinion, is reduction in the deterioration of heart function, which is certainly different from improvement in deteriorated heart function. What do you think?

Dr Yerebakan. This is extremely interesting. I am convinced by this simple surgical method, because it has already been used clinically, and I was looking for mechanisms, as you correctly raise, possible mechanisms to explain the effect.

Just shortly for the sake of time, it might be a leftward shift of the interventricular septum with reduction of the mitral regurgitation. We didn't have a lot of significant mitral regurgitation in our animals, and I don't think this would be the leading mechanism in these experiments. Leftward shift of the interventricular septum leads to a reduction of left ventricular dimensions and prevention of a deterioration of left ventricular function before an irreversible loop of dilation with following occurs. Additionally, the preload reduction of the left ventricle might have contributed to favorable hemodynamics after pulmonary artery banding.

Second mechanism might be a regenerative pathway following PAB with stimulation of resident or peripheral stem cells with induction of a reverse remodeling of the left ventricle. The time period in this experiment might be too short to speculate that this is the mechanism.

And the third might be the so-called Anrep effect, which was described in 1912. That implies an afterload increase of the left or right ventricle with better contractility.

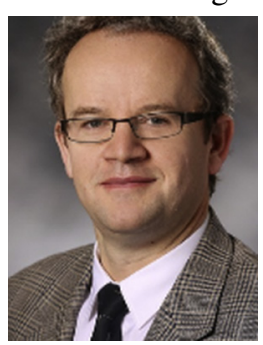

Dr Tomasz Timek (Grand Rapids, Mich). We would like to cut you off here a bit, and I appreciate the discussion, but before we let you off the hook, are you trading left ventricular dysfunction for right ventricular dysfunction, yes or no?

Dr Yerebakan. We are treating left ventricular dysfunction.

Dr Timek. What happens to the right side?

Dr Yerebakan. I cannot answer with the data what happens to the right side, but I know from my previous experiments the chronic afterload increase of the right ventricle leads to fibrosis. But in these patients clinically, we partially de-band the patients at midterm follow-up to prevent right ventricular dysfunction. 


\section{APPENDIX E1. MACROSCOPIC INVESTIGATION}

Hearts including the epicardium and pericardium were visually inspected for any abnormalities. The heart was weighted, and the following dimensions were measured: outer circumference, height of auricular and atrial facies, size of the left and right atria (length $\times$ width $\times$ height), and extension of the left and right ventricle (length $\times$ width).

The vessels were also prepared and cut to assess the maximal and minimal outer and inner diameters of the brachiocephalic artery, as well as outer and inner diameters of the right pulmonary artery, superior (cranial) vena cava, and inferior (caudal) vena cava. The aorta was assessed at 3 different locations: its origin, in the middle $(2 \mathrm{~cm}$ from the origin), and at the cut ( $\sim 4 \mathrm{~cm}$ from origin). Maximal and minimal outer and inner aortic diameters were measured. The same procedure was performed for the pulmonary trunk. Maximal and minimal outer and inner diameters were also measured for the left coronary artery. The left coronary artery is more prominent in ruminants than the right coronary artery for which outer and inner diameters were also measured.

The heart was then opened, and maximal and minimal atrial wall widths as well as lengths of the left and right atrioventricular ring were measured. The maximal dimensions (length, width, height) of the sinus venarum cavarum were measured on the right atrium. Atria were dissected, and the weight of both ventricles including the septum was measured. Ventricles and septum were then separated. Weight, inner dimensions (length $\times$ width), and wall widths were measured. The latter were measured on the inflow and outflow areas of each ventricle at the heart's base, middle, and apex, respectively. Dimensions of major papillary muscles (length, width, height) and septal wall width on the base, middle, and apex were also assessed.

All investigations were carried out by a board-certified veterinary pathologist. Because a stenosis was clearly visible on the pulmonary trunk of banded animals, but absent in sham animals, macroscopic assessment could not be carried out in a blinded fashion.

\section{MICROSCOPIC INVESTIGATION OF INFLAMMATORY CELL LOAD AND FIBROSIS}

Small tissue blocks were gained from 2 locations in the left ventricle, that is, from the facies auricularis close to the subauricular (location A) and subatrial papillary muscle (location B), respectively. Tissue samples were embedded in Paraplast (Vogel, Gießen, Germany) using a Hypercenter XP automated embedding device (Shandon, Frankfurt, Germany). According to the manufacturer's instructions, the tissue was cut into $4-\mu \mathrm{m}$ slices using a sledge microtome (Reichert-Jung, Vienna, Austria). Slices were stained with hematoxylin-eosin (immune cell invasion) or Picrosirius red (fibrosis) according to standard procedures. Images were taken using a DP-26 camera connected to a BH-2 microscope (both Olympus, Hamburg, Germany) and stored digitally. All analyses were performed using the cellSens Dimension software (Olympus). Nine randomly chosen high-power field (HPFs) images were assessed. Three HPFs each from an area close to the epicardium, central, and close to the endocardium were evaluated.

Inflammatory cell load (mononuclear cells and neutrophils) was measured by counting the number of cells in each HPF and categorized using the following score: 0 (0-5 cells/HPF), 0.5 (6-25 cells), 1.0 (26-45 cells), 1.5 (46-65 cells), 2.0 (66-85 cells), 2.5 (86-105 cells), and 3.0 (>105 cells). For fibrosis assessment, images were optimized for contrast using the embedded software tools. Collagenous fibers (fibrosis) appear in bright red to red, depending on their disposition density in Picrosirius red staining. Therefore, color variability was compensated by adjusting the hue, saturation, and value threshold of each image. Fibrosis area $\left(\mu \mathrm{m}^{2} / \mathrm{HPF}\right)$ was then automatically assessed by the software. Minimal size of included objects was set to 100 pixels. Inflammatory cell scores and fibrotic area were averaged for the 3 HPFs observed in each area. Microscopic investigations were carried out by a board-certified veterinary pathologist who was blinded to the experimental groups. 

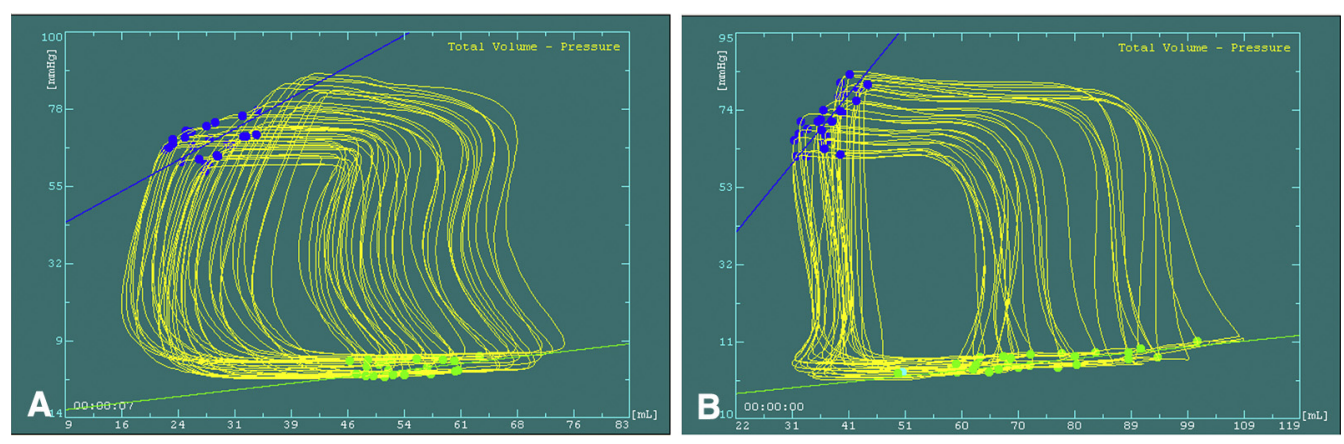

FIGURE E1. Representative PV loop recordings from the sham (A) and cPAB (B) groups in comparison. The volume is depicted in the $\mathrm{x}$-axis, and the pressure is depicted in the y-axis. Each of the loops represent 1 cardiac cycle during preload reduction maneuvers. The slopes of end-diastolic (Eed) and end-systolic (Ees) PV relations can be calculated from the green and blue curves, respectively.

TABLE E1. Macroscopic dimensions of the right heart after explantation

\begin{tabular}{|c|c|c|c|}
\hline & Sham & CPAB & $P$ value \\
\hline \multicolumn{4}{|l|}{ Atrium } \\
\hline Atrial volume (external dimensions, $\mathrm{cm}^{3}$ ) & $43.63 \pm 9.26$ & $62.04 \pm 36.34$ & .17 \\
\hline Atrial volume (sinus venarum cavarum, $\mathrm{cm}^{3}$ ) & $1.56 \pm 0.37$ & $2.62 \pm 1.90$ & .14 \\
\hline Minimal atrial wall width $(\mathrm{cm})$ & $0.10 \pm 0.00$ & $0.17 \pm 0.05$ & $<.01$ \\
\hline Maximal atrial wall width $(\mathrm{cm})$ & $0.41 \pm 0.09$ & $0.53 \pm 0.07$ & $<.05$ \\
\hline Length atrioventricular ring $(\mathrm{cm})$ & $8.61 \pm 1.25$ & $9.44 \pm 0.90$ & .13 \\
\hline \multicolumn{4}{|l|}{ Ventricle, inflow area } \\
\hline Wall width, basis $(\mathrm{cm})$ & $0.50 \pm 0.05$ & $0.59 \pm 0.19$ & .12 \\
\hline Wall width, center $(\mathrm{cm})$ & $0.47 \pm 0.10$ & $0.60 \pm 0.17$ & $<.05$ \\
\hline Wall width, apex $(\mathrm{cm})$ & $0.39 \pm 0.12$ & $0.47 \pm 0.12$ & .19 \\
\hline \multicolumn{4}{|l|}{ Ventricle, outflow area } \\
\hline Wall width, basis $(\mathrm{cm})$ & $0.47 \pm 0.11$ & $0.53 \pm 0.10$ & .20 \\
\hline Wall width, center $(\mathrm{cm})$ & $0.47 \pm 0.11$ & $0.51 \pm 0.19$ & .56 \\
\hline Wall width, apex $(\mathrm{cm})$ & $0.31 \pm 0.09$ & $0.48 \pm 0.25$ & .09 \\
\hline \multicolumn{4}{|l|}{ Large papillary muscle } \\
\hline Volume $\left(\mathrm{cm}^{3}\right)$ & $0.59 \pm 0.27$ & $1.04 \pm 0.58$ & $<.05$ \\
\hline
\end{tabular}

Bold indicates $P<.05 . c P A B$, Central pulmonary artery banding. 
TABLE E2. Macroscopic dimensions of the left heart after explantation

\begin{tabular}{|c|c|c|c|}
\hline & Sham & cPAB & $P$ value \\
\hline \multicolumn{4}{|l|}{ Atrium } \\
\hline Atrial volume (external dimensions, $\mathrm{cm}^{3}$ ) & $66.3 \pm 56.2$ & $64.9 \pm 18.8$ & .95 \\
\hline Minimal atrial wall width $(\mathrm{cm})$ & $0.18 \pm 0.07$ & $0.18 \pm 0.07$ & 1.00 \\
\hline Maximal atrial wall width $(\mathrm{cm})$ & $0.51 \pm 0.14$ & $0.54 \pm 0.07$ & .53 \\
\hline Length atrioventricular ring $(\mathrm{cm})$ & $7.21 \pm 0.83$ & $7.78 \pm 0.89$ & .18 \\
\hline \multicolumn{4}{|l|}{ Ventricle, inflow area } \\
\hline Wall width, basis $(\mathrm{cm})$ & $1.17 \pm 0.17$ & $1.19 \pm 0.18$ & .79 \\
\hline Wall width, center $(\mathrm{cm})$ & $1.03 \pm 0.15$ & $0.99 \pm 0.14$ & .52 \\
\hline Wall width, apex $(\mathrm{cm})$ & $0.71 \pm 0.20$ & $0.79 \pm 0.17$ & .38 \\
\hline \multicolumn{4}{|l|}{ Ventricle, outflow area } \\
\hline Wall width, basis (cm) & $1.20 \pm 0.28$ & $1.18 \pm 0.17$ & .84 \\
\hline Wall width, center $(\mathrm{cm})$ & $0.99 \pm 0.18$ & $1.06 \pm 0.24$ & .51 \\
\hline Wall width, apex $(\mathrm{cm})$ & $0.92 \pm 0.18$ & $0.90 \pm 0.15$ & .78 \\
\hline Minimal wall width (overall, $\mathrm{cm}$ ) & $0.48 \pm 0.14$ & $0.47 \pm 0.16$ & .88 \\
\hline \multicolumn{4}{|l|}{ Papillary muscle volumes } \\
\hline Musculus papillaris subauricularis $\left(\mathrm{cm}^{3}\right)$ & $2.10 \pm 0.93$ & $2.58 \pm 0.96$ & .30 \\
\hline M papillaris subatrialis $\left(\mathrm{cm}^{3}\right)$ & $2.43 \pm 1.46$ & $2.76 \pm 1.11$ & .61 \\
\hline
\end{tabular}

TABLE E3. Macroscopic dimensions of the septum

\begin{tabular}{lccc}
\hline & Sham & cPAB & $\boldsymbol{P}$ value \\
\hline Wall width basis $(\mathrm{cm})$ & $1.19 \pm 0.28$ & $1.21 \pm 0.32$ & .88 \\
Wall width center $(\mathrm{cm})$ & $1.02 \pm 0.11$ & $1.18 \pm 0.32$ & .20 \\
Wall width apex $(\mathrm{cm})$ & $0.98 \pm 0.04$ & $1.09 \pm 0.43$ & .46 \\
\hline
\end{tabular}

$C P A B$, Central pulmonary artery banding. 
TABLE E4. Macroscopic dimensions of large vessels

\begin{tabular}{|c|c|c|c|}
\hline & Sham & сPAB & $P$ value \\
\hline \multicolumn{4}{|l|}{ Truncus pulmonalis } \\
\hline Inner diameter at basis $\min / \max (\mathrm{cm})$ & $1.08 \pm 0.23 / 1.23 \pm 0.23$ & $1.27 \pm 0.23 / \mathbf{1 . 6 9} \pm \mathbf{0 . 2 7}$ & $.09 / .01$ \\
\hline Outer diameter at basis $\min / \max (\mathrm{cm})$ & $1.53 \pm 0.23 / 1.70 \pm 0.22$ & $1.84 \pm 0.29 / 2.28 \pm 0.31$ & $<.05 /<.01$ \\
\hline Inner diameter at center min/max $(\mathrm{cm})$ & $1.00 \pm 0.13 / 1.21 \pm 0.15$ & $0.74 \pm 0.21 / 0.98 \pm 0.20$ & $<.01 / .02$ \\
\hline Outer diameter at center $\min / \max (\mathrm{cm})$ & $1.50 \pm 0.12 / 1.73 \pm 0.12$ & $1.51 \pm 0.40 / 1.89 \pm 0.73$ & $.94 / .55$ \\
\hline Inner diameter at top* $\min / \max (\mathrm{cm})$ & $0.99 \pm 0.20 / 1.18 \pm 0.16$ & $\mathbf{0 . 7 4} \pm \mathbf{0 . 2 5} / 1.12 \pm 0.32$ & $<.05 / .65$ \\
\hline Outer diameter at top* $\min / \max (\mathrm{cm})$ & $1.40 \pm 0.21 / 1.58 \pm 0.20$ & $1.19 \pm 0.33 / 1.53 \pm 0.35$ & $.13 / .77$ \\
\hline \multicolumn{4}{|l|}{ Aorta } \\
\hline Inner diameter at basis $\min / \max (\mathrm{cm})$ & $0.74 \pm 0.15 / 1.30 \pm 0.23$ & $0.78 \pm 0.28 / 1.23 / 0.14$ & $.76 / .48$ \\
\hline Outer diameter at basis $\min / \max (\mathrm{cm})$ & $1.33 \pm 0.16 / 1.78 \pm 0.20$ & $1.37 \pm 0.21 / 1.77 \pm 0.12$ & $.71 / .89$ \\
\hline Inner diameter at center min/max $(\mathrm{cm})$ & $0.80 \pm 0.10 / 1.32 \pm 0.17$ & $0.78 \pm 0.24 / 1.26 \pm 0.12$ & $.81 / .36$ \\
\hline Outer diameter at center $\min / \max (\mathrm{cm})$ & $1.27 \pm 0.14 / 1.74 \pm 0.19$ & $1.37 \pm 0.18 / 1.71 \pm 0.09$ & $.21 / .65$ \\
\hline Inner diameter at top $\nmid \min / \max (\mathrm{cm})$ & $0.79 \pm 0.12 / 1.20 \pm 0.24$ & $0.73 \pm 0.19 / 1.22 \pm 0.11$ & $.46 / .80$ \\
\hline Outer diameter at top $\dagger \min / \max (\mathrm{cm})$ & $1.30 \pm 0.20 / 1.66 \pm 0.24$ & $1.32 \pm 0.15 / 1.72 \pm 0.13$ & $.80 / .48$ \\
\hline \multicolumn{4}{|l|}{ Truncus brachiocephalicus } \\
\hline Inner diameter $\min / \max (\mathrm{cm})$ & $0.59 \pm 0.11 / 0.87 \pm 0.17$ & $\mathbf{0 . 4 3} \pm \mathbf{0 . 1 0} / 0.93 \pm 0.21$ & $<.01 / .47$ \\
\hline Outer diameter $\min / \max (\mathrm{cm})$ & $1.01 \pm 0.12 / 1.34 \pm 0.20$ & $\mathbf{0 . 8 6} \pm \mathbf{0 . 1 6} / 1.33 \pm 0.20$ & $<.05 / .91$ \\
\hline \multicolumn{4}{|l|}{ Arteria pulmonalis dexta } \\
\hline Inner/outer diameter $(\mathrm{cm})$ & $0.69 \pm 0.16 / 0.93 \pm 0.16$ & $0.69 \pm 0.20 / 0.96 \pm 0.22$ & $1.00 / .81$ \\
\hline \multicolumn{4}{|l|}{ Vena cava caudalis } \\
\hline Inner/outer diameter $(\mathrm{cm})$ & $1.02 \pm 0.25 / 1.23 \pm 0.27$ & $1.14 \pm 0.31 / 1.34 \pm 0.31$ & $.37 / .43$ \\
\hline \multicolumn{4}{|l|}{ V. cava cranialis } \\
\hline Inner/outer diameter $(\mathrm{cm})$ & $1.18 \pm 0.29 / 1.40 \pm 0.30$ & $1.06 \pm 0.27 / 1.28 \pm 0.29$ & $.38 / .39$ \\
\hline
\end{tabular}

Bold indicates $P<.05 . c P A B$, Central pulmonary artery banding. *Before right pulmonary artery. $\dagger$ Aorta was cut $4 \mathrm{~cm}$ from origin. 